\title{
Identification of keygenes, miRNAs and miRNA-mRNA regulatory pathways for chemotherapy resistance in ovarian cancer
}

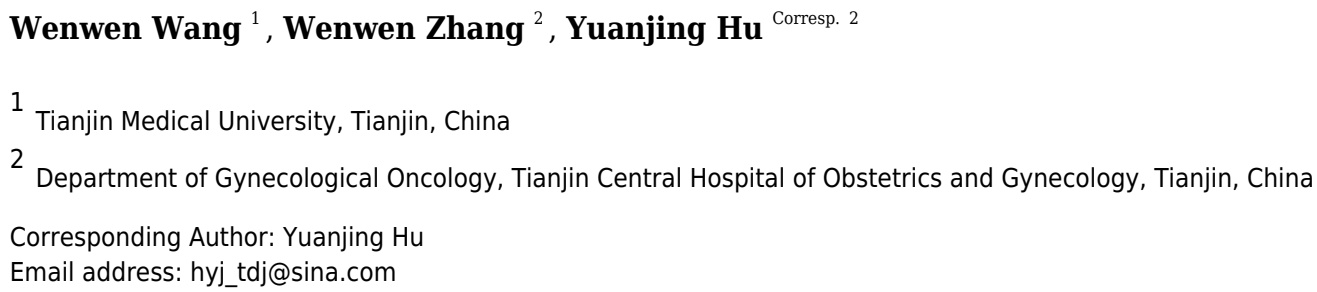

Background: Chemotherapy resistance, especially platinum resistance, is the main cause of poor prognosis of ovarian cancer. It is of great urgency to find molecular markers and mechanism related to platinum resistance in ovarian cancer.

Methods: One mRNA dataset (GSE28739) and one miRNA dataset (GSE25202) were acquired from Gene Expression Omnibus (GEO) database. The GEO2R tool was used to screen out differentially expressed genes (DEGs) and differentially expressed miRNAs (DEmiRNAs) between platinum-resistant and platinum-sensitive ovarian cancer patients. Gene Ontology (GO) function and Kyoto Encyclopedia of Genes and Genomes (KEGG) pathway enrichment analysis for DEGs were performed using the DAVID to present the most visibly enriched pathways. Protein-protein interaction (PPI) of these DEGs was constructed based on the information of the STRING database. Hub genes related to platinum resistance were visualized by Cytoscape software. Then, we chose seven interested hub genes to further validate using qRT-PCR in A2780 ovarian cancer cell lines. And, at last, the TF-miRNAtarget genes regulatory network was predicted and constructed using miRNet software. Results: A total of 63 upregulated DEGs, 124 downregulated DEGs, 4 upregulated miRNAs and 6 downregulated miRNAs were identified. From the PPI network, the top 10 hub genes were identified, which were associated with platinum resistance. Our further qRT-PCR showed that seven hub genes (BUB1, KIF2C, NUP43, NDC80, NUF2, CCNB2 and CENPN) were differentially expressed in platinum-resistant ovarian cancer cells. Furthermore, the upstream transcription factors (TF) for upregulated DE-miRNAs were SMAD4, NFKB1, SMAD3, TP53 and HNF4A. Three overlapping downstream target genes (KIF2C, STAT3 and BUB1) were identified by miRNet, which was regulated by hsa-miR-494.

Conclusions: The TF-miRNA-mRNA regulatory pairs, that is TF (SMAD4, NFKB1 and SMAD3)-miR-494-target genes (KIF2C, STAT3 and BUB1), were established. In conclusion, the present study is of great significance to find the key genes of platinum resistance in 
ovarian cancer. Further study is needed to identify the mechanism of these genes in ovarian cancer. 


\section{Identification of key genes, miRNAs and miRNA-mRNA regulatory pathways}

2

3

$4 \quad 1$ Tianjin Medical University, Tianjin, China

52 Department of Gynecological Oncology, Tianjin Central Hospital of Obstetrics and

6 Gynecology, Tianjin, China

7 *Corresponding Author:

8 Hu yuanjing

9 156th Sanma Road, Tianjin, 300199, China

12 Abstract

\section{for chemotherapy resistance in ovarian cancer}

Wang Wenwen ${ }^{1}$, Zhang Wenwen ${ }^{2}$, Hu yuanjing ${ }^{2 *}$

Email address:Correspondence: hyj_tdj@sina.com
Background: Chemotherapy resistance, especially platinum resistance, is the main cause of poor prognosis of ovarian cancer. It is of great urgency to find molecular markers and mechanism related to platinum resistance in ovarian cancer.

Methods: One mRNA dataset (GSE28739) and one miRNA dataset (GSE25202) were acquired from Gene Expression Omnibus (GEO) database. The GEO2R tool was used to screen out differentially expressed genes (DEGs) and differentially expressed miRNAs (DE-miRNAs) between platinum-resistant and platinum-sensitive ovarian cancer patients. Gene Ontology (GO) function and Kyoto Encyclopedia of Genes and Genomes (KEGG) pathway enrichment analysis for DEGs were performed using the DAVID to present the most visibly enriched pathways. 
22 Protein-protein interaction (PPI) of these DEGs was constructed based on the information of the

23 STRING database. Hub genes related to platinum resistance were visualized by Cytoscape

24 software. Then, we chose seven interested hub genes to further validate using qRT-PCR in

25 A2780 ovarian cancer cell lines. And, at last, the TF-miRNA-target genes regulatory network

26 was predicted and constructed using miRNet software.

27 Results: A total of 63 upregulated DEGs, 124 downregulated DEGs, 4 upregulated miRNAs and

6 downregulated miRNAs were identified. From the PPI network, the top 10 hub genes were identified, which were associated with platinum resistance. Our further qRT-PCR showed that seven hub genes (BUB1, KIF2C, NUP43, NDC80, NUF2, CCNB2 and CENPN) were differentially expressed in platinum-resistant ovarian cancer cells. Furthermore, the upstream transcription factors (TF) for upregulated DE-miRNAs were SMAD4, NFKB1, SMAD3, TP53 and HNF4A. Three overlapping downstream target genes (KIF2C, STAT3 and BUB1) were identified by miRNet, which was regulated by hsa-miR-494.

Conclusions: The TF-miRNA-mRNA regulatory pairs, that is TF (SMAD4, NFKB1 and SMAD3)-miR-494-target genes (KIF2C, STAT3 and BUB1), were established. In conclusion, the present study is of great significance to find the key genes of platinum resistance in ovarian cancer. Further study is needed to identify the mechanism of these genes in ovarian cancer.

Keywords: ovarian cancer, platinum resistance, hub genes, mRNA, miRNA, expression profiling data, bioinformatics analysis

\section{Introduction}

42 Epithelial ovarian cancer (EOC) is the third highest incidence of carcinoma in the female 
43 reproductive system with the highest mortality rate. The standard of treatment of ovarian cancer

44 is surgical resection followed by platinum-based chemotherapy(Armstrong et al. 2021).

45 Platinum compounds bind to DNA and create intra-strand or inter-strand DNA crosslinks that

46 induce DNA damage(Wang and Lippard 2005). However, chemotherapy resistance, especially

47 platinum resistance, leads to poor prognosis of ovarian cancer patients(Agarwal and Kaye 2003).

48 However, the molecular mechanisms of platinum resistance in ovarian cancer remain unclear.

49 Therefore, a comprehensive understanding of the mechanism of platinum resistance is the key to

50 improve outcomes of women with EOC.

51 Currently, despite more and more studies use bioinformatics methods to analyze differentially

52 expressed genes and miRNAs in ovarian cancer(Yang et al. 2020; Zheng et al. 2019; Cao et al.

53 2019; Feng et al. 2019; Gong, Lin, and Yuan 2020; Li and Li 2019; Lu et al. 2020; Zhang et al.

54 2019), to our knowledge, a systematic and comprehensive analysis of miRNA-mRNA regulatory

55 network based on clinical samples of cisplatin resistance in ovarian cancer is still absent. In this

56 study, we tried to construct and analyze the transcription factors-miRNA-target genes regulatory

57 network using bioinformatics methods in order to find the potential mechanism and markers of

58 platinum resistance in ovarian cancer patients. To detect the DEGs and DE-miRNAs between

59 platinum-resistant and platinum-sensitive ovarian cancer patients, we used the datasets

60 downloaded from the Gene Expression Omnibus (GEO) database. Gene Ontology (GO)

61 functional annotation analysis and Kyoto Encyclopedia of Genes and Genomes (KEGG)

62 pathway enrichment analysis were performed using the DAVID for the screened DEGs. We

63 established a protein-protein interaction (PPI) network to identify hub genes related to platinum 
64 resistance. Then, the analyzed results were verified by using quantitative reverse transcription

65 polymerase chain reaction (qRT-PCR) assay in A2780 ovarian cancer cells. At last, the TF-

66 miRNA-target genes regulatory network was predicted and constructed using miRNet software

67 and Cytoscape.

68 Materials \& Methods

\section{Data source}

The gene expression datasets analyzed in this study were obtained from the GEO database (https://www.ncbi.nlm.nih.gov/geo/). GSE28739 was established on the platform of GPL7264 (Agilent-012097 Human 1A Microarray (V2)). There were 25 patients in this data set, including 15 chemo-resistant serous EOC patients [time to recurrence (TTR) $\leqslant 6$ months] and 10 chemosensitive serous EOC patients (TTR $\geqslant 30$ months). 30 chemo-resistant serous EOC samples and 20 chemo-sensitive serous EOC samples were analyzed to screen DEGs.

GSE 25202 was based on the platform of GPL8179 (Illumina Human v2 MicroRNA expression beadchip). The dataset contained 55 surgical specimens including 30 early relapsing patients (optimally debulked patients with TTR $<12$ months and sub-optimally debulked patients with TTR $<6$ months) and 25 late relapsing patients (optimally debulked patients with TTR $>36$ months and sub-optimally debulked patients with TTR $>12$ months). All of the data were available for free online.

Identification of DEGs and DE-miRNAs

GEO online analysis tool GEO2R (https:/www.ncbi.nlm.nih.gov/geo/geo2r/) was used. Genes 
85

86

DEGs. Adjusted $\mathrm{p}$ value $<0.05$ and $\mid \log _{2}$ Fold Change $\mid \geqslant 1.5$ were set as the thresholds for identifying DE-miRNAs.

\section{GO functional enrichment analysis of DEGs}

GO functional analysis is a common method to analyze the functions of DEGs, which can be classified into biological process (BP), molecular function (MF), and cellular component (CC). GO functional analysis was carried out utilizing DAVID online tool (https://david.ncifcrf.gov/). $P$ value $<0.05$ and gene counts $\geqq 3$ were considered as significant.

\section{KEGG pathway analysis of DEGs}

KEGG pathway analysis is a widely used database to explore potential relationships among these DEGs. It was also carried out by DAVID online tool. $\mathrm{P}$ value $<0.05$ and gene counts $\geqq 5$ were considered as significant.

\section{PPI network construction and hub gene identification}

The online Search Tool for the Retrieval of Interacting Genes (STRING) database is used to construct PPI network among DEGs (http://string-db.org/). Subsequently, the PPI network was visualized by Cytoscape software (www.cytoscape.org/). One plugin in Cytoscape, CytoHubba, was used to screen hub genes, which were evaluated by connectivity degree of each protein node. The top ten genes were identified as hub genes in the study.

Prediction of Potential Transcription Factors and Target Genes of DE-miRNAs The upstream transcription factors (TF) and the target genes of the DE-miRNAs were predicted using miRNet database (https://www.mirnet.ca/). The TF-miRNA-target genes regulatory network was depicted and visualized using Cytoscape software (www.cytoscape.org/). 
106

107

108

109

110

111

112

113

114

115

116

117

118

119

120

121

122

123

124

125

126

\section{Drug preparation}

Cis-Diammineplatinum dichloride (Cis-platinum) was obtained from Aladdin (Shanghai, China) and dissolved in Dimethyl sulfoxide (DMSO) at a final concentration of $2 \mathrm{mg} / \mathrm{ml}$. Aliquots were stored at $-20^{\circ} \mathrm{C}$ and thawed immediately before use.

\section{Cell lines}

A2780 and its cis-platinum resistant cell lines were gifts from Professor Li Bin from National Cancer Institute, Cancer Hospital, Chinese Academy of Medical Sciences, Peking Union Medical College. A2780 cells were maintained in RPMI-1640 containing 10\% fetal bovine serum under atmosphere of $5 \% \mathrm{CO} 2$ with humidity at $37^{\circ} \mathrm{C}$. A2780 cis-platinum resistant cell line was cultured in RPMI-1640 containing cis-platinum with the concentration of $200 \mathrm{ng} / \mathrm{ml}$ and $10 \%$ fetal bovine serum under atmosphere of $5 \% \mathrm{CO} 2$ with humidity at $37^{\circ} \mathrm{C}$.

\section{Quantitative RT-PCR}

Total RNAs from cell lines were extracted using TRIzol Reagent (Ambion, USA) according to the manufacturer's instructions. Total RNA was then reverse transcribed into cDNA in a final volume of $40 \mu \mathrm{L}$ using M5 Super qPCR RT kit (Mei5 Biotechonlogy, Beijing, China). Then qPCR amplifications were carried out in the $20 \mu \mathrm{L}$ reaction mixtures with $2 x$ SYBR Green qPCR

Master Mix (Bimake, USA). The program was as follows: $50^{\circ} \mathrm{C}$ for 2 mins, $95^{\circ} \mathrm{C}$ for $10 \mathrm{mins}, 95^{\circ} \mathrm{C}$ for $15 \mathrm{~s}$ and $60^{\circ} \mathrm{C}$ for $1 \mathrm{~min}$ for 45 cycles. The primers were purchased from Sangon Biotech Co. (Shanghai, China). Expression of GAPDH was used as the internal control, and the nucleotide sequences for primers are listed in Table 1 . The relative mRNA levels were analyzed by the $2^{\wedge} \Delta \Delta \mathrm{CT}$ method. Each experiment was performed in triplicate. 
127

128

129

130

131

132

133

134

135

136

137

\section{Statistical analysis}

Statistical analysis was performed using SPSS 22.0. Student's t test was used to assess the significance among groups. GraphPad Prism 8.0.2 was used for plotting graphs. $\mathrm{p}<0.05$ was considered to be a significant difference.

\section{Results}

\section{Identification of DEGs and DE-miRNAs}

Based on GEO2R analysis, a total of 187 DEGs (63 upregulated genes and 124 downregulated genes) were identified based on the criteria of adjusted $p$ value $<0.05$ and $\mid \log _{2}$ Fold Change $\mid \geqslant 0.5$. The volcano plot of DEGs is shown in Figure 1. All of the upregulated and downregulated DEGs are shown in Table 2. In miRNA datasets GES25202, a total of 10 DE-miRNAs (4 upregulated miRNAs and 6 downregulated miRNAs) were detected, which was shown in Table 3. The volcano plot of miRNAs is presented in Figure 2.

\section{Gene Ontology Term Enrichment Analysis of DEGs}

The results of GO functional analysis are shown in Figure 3. Biological processes (BP) contained nucleosome assembly, CENP-A containing nucleosome assembly, telomere organization, DNAtemplated transcription, chromatin, silencing at rDNA, protein heterotetramerization, betacatenin-TCF complex assembly, negative regulation of gene expression, positive regulation of gene expression, oxygen transport, double-strand break repair via nonhomologous end joining and sister chromatid cohesion. For the cellular component category (CC), the DEGs were enriched in nucleosome, nuclear chromosome, hemoglobin complex, condensed chromosome kinetochore, haptoglobin-hemoglobin complex, Ndc80 complex, extracellular exosome. For the 
148 molecular function category (MF), the DEGs were correlated with protein binding, haptoglobin

149 binding, oxygen transporter activity.

150

151

152

153

154

155

156

157

158

159

160

161

162

163

164

165

166

167

168

\section{Kyoto Encyclopedia of Genes and Genomes Pathway Analysis of DEGs}

The results of KEGG pathway enrichment are shown in Figure 4. The significant KEGG pathways of DEGs included systemic lupus erythematosus; viral carcinogenesis; alcoholism; malaria; African trypanosomiasis; non-alcoholic fatty liver disease (NAFLD); tuberculosis and mismatch repair.

\section{Protein-Protein Interaction Network and hub gene identification}

The PPI network of the DEGs was constructed with STRING tools. The result of PPI network was displayed in Figure 5. The PPI network included 184 nodes and 108 edges, and the PPI enrichment P-value was $1.11 \mathrm{e}-16$. We also structured the PPI network with DEGs and showed hub genes. The top ten genes (NUF2, CCNB2, CENPN, HIST1H2BE, BUB1, NDC80, NUP43, KIF2C, STAT3 and HIST1H2BC), with the highest degree scores of connectivity in the PPI network were identified as hub genes (Table 4; Figure 6; Figure 7).

\section{The expression of hub genes in cell lines}

Most of the hub genes are related to the regulation of cell cycle. And we choose seven interested hub genes to validate in A2780 cell lines by qRT-PCR. The results of relative expression of these seven genes are listed in Figure 8.

\section{Prediction of Transcription Factors of DE-miRNAs}

In our study, we used miRNet database to predict upstream transcription factors of DE-miRNAs. As all of the 10 hub genes identified in the study were downregulated, so we predicted the 
169

170

171

172

173

174

175

176

177

178

179

180

181

182

183

184

185

transcription factors of upregulated miRNAs, which were presented in Figure 9. For upregulated

DE-miRNAs, the upstream transcription factors were SMAD4, NFKB1, SMAD3, TP53 and

HNF4A.

\section{Construction of miRNA-target gene regulatory network}

The miRNet online tool predicted 4056 target genes for the 4 upregulated DE-miRNAs. And then, KIF2C, STAT3 and BUB1 among the predicted target genes also belonged to the 10 hub genes of GSE 28739, which was regulated by hsa-miR-494. The miRNA-target gene regulatory network was constructed and visualized in Figure 10 using Cytoscape.

\section{Discussion}

Clinically, platinum sensitivity is defined as patients with a platinum-free interval (PFI) of greater than 6 months and platinum resistance is defined as those with a PFI of less than 6 months(Gore et al. 1990). The reason for platinum resistance in epithelial ovarian cancer is complex. It may include intracellular drug inactivation, reduced intracellular drug accumulation, increased DNA repair and inhibition of cell death(Freimund et al. 2018). Currently, mechanisms of platinum resistance in ovarian cancer have been extensively studied, but mostly are in ovarian cancer cell lines(Dorayappan et al. 2018; Mo et al. 2013; Tassi et al. 2019; Zhang, Chen, et al. 2018; Huo et al. 2016; Zhang et al. 2020). Hence, considering the heterogeneity of patients, it is crucial to explore the mechanism of platinum resistance in ovarian cancer patients.

MiRNAs are a kind of noncoding RNAs, which bind with targeted mRNAs to regulate mRNA expression. They play a pivotal role in RNA silencing and post-transcriptional regulation of gene expression(Lee and Dutta 2009). However, there have been few studies on miRNAs involved in 
190

191

192

193

194

195

196

197

198

199

200

201

202

203

204

205

206

207

208

209

210

platinum resistance for ovarian cancer. It may help us identify new markers, specific pathways and targeted therapies for platinum-resistant EOC.

In the article, DEGs between platinum-resistant and platinum-sensitive ovarian cancer patients were screened out based on gene expression profiling data from the GEO database. Totally, 63 upregulated DEGs and 124 downregulated DEGs were identified. GO analysis showed that these DEGs were associated with the BP terms, such as nucleosome assembly, CENP-A containing nucleosome assembly, telomere organization, DNA-templated transcription, chromatin, silencing at rDNA, protein heterotetramerization, beta-catenin-TCF complex assembly, negative regulation of gene expression, positive regulation of gene expression, oxygen transport, doublestrand break repair via nonhomologous end joining and sister chromatid cohesion. The pathways significantly enriched in KEGG analysis contained systemic lupus erythematosus; viral carcinogenesis; alcoholism; malaria; African trypanosomiasis; non-alcoholic fatty liver disease (NAFLD); tuberculosis and mismatch repair. A PPI network was constructed to investigate the interrelationship of the DEGs, and ten hub genes were identified, including NUF2, CCNB2, CENPN, NDC80, HIST1H2BE, HIST1H2BC, BUB1, NUP43, KIF2C and STAT3. All of these genes were downregulated in platinum resistant ovarian cancer patients.

As all of the 10 hub genes identified in our study were downregulated, 4 upregulated miRNAs (miR-656, miR-655, miR-379*, miR-494) were finally selected. After conducting a combined analysis of DEGs and target genes of DE-miRNAs, 3 overlapping target genes (KIF2C, STAT3 and BUB1) were further screened, which was regulated by hsa-miR-494. And in our study, we predicted the upstream transcription factors of hsa-miR-494 were SMAD4, NFKB1, SMAD3. 
211 Previous studies have shown that microRNA-494 exerts its effect in cell proliferation, migration,

212 invasion and chemosensitivity in gastric cancer, colorectal cancer, pancreatic cancer, prostate

213 cancer and so on (Wan, Cheng, and Zhang 2019; Ghorbanhosseini et al. 2019; Peng et al. 2018; He et al. 2018; Xu et al. 2018; Zhang,

Guo, et al. 2018; Shen et al. 2014). And one study about ovarian cancer concluded that microRNA-494 is a

potential prognostic marker and inhibits cellular proliferation, migration and invasion by

targeting SIRT1(Yang et al. 2017).

Among the overlapping three target genes, STAT3 is the most widely studied. STAT3, that is signal transducer and activator of transcription factor 3, relates not only to the proliferation, invasion, metastasis, apoptosis of ovarian cancer cells, but also chemotherapy resistance(Zhong et al. 2021; Yu et al. 2020; Liu et al. 2021; Pan et al. 2020).

BUB1 (budding uninhibited by benzimidazole 1) is a mitotic checkpoint protein that is

overexpressed in various types of cancer, including breast cancer, gastric cancer, pancreatic cancer and so on(Han et al. 2015; Piao et al. 2019; Bai et al. 2019). Its high expression correlates with a poor clinical prognosis. In a bioinformatics study, BUB1 was reported to be overexpressed in ovarian cancer compared to normal ovarian tissue(Yang et al. 2020). Another study confirmed that BUB1 was widely expressed in primary and metastatic ovarian cancer at the level of mRNA and protein(Davidson et al. 2014). In our study, we find a new mechanism of BUB1. It may be correlated with cisplatin resistance in ovarian cancer. important roles in intracellular transport of organelles and maintenance of spindle assembly 
232 Up-regulation of KIF2C has been documented in multiple human cancers, such as breast cancer, 233 gastric cancer, colorectal cancer, glioma, liver cancer and non-small cell lung cancer(Wei et al.

234 2020; Gan et al. 2019; Li et al. 2020; Bie et al. 2012; Ishikawa et al. 2008; Nakamura et al. 2007).

235 One study reports that KIF2C may be involved in the development of paclitaxel resistance in 236 ovarian cancer(Zhao et al. 2014). In our study, the expression of KIF2C was lower in platinum237 resistant ovarian cancer cell lines.

238 Although we constructed a potential TF-miRNA-mRNA regulatory network in this study, 239 some limitations still exist. First, the dataset GSE28739 in this study included a small sample 240 size of data. Second, lack of experimental validation of our predicted TF-miRNA-mRNA 241 regulatory network is another limitation of the study. Therefore, experimental studies should be 242 executed to validate the TF-miRNA-mRNA regulatory network in the future. And the 243 mechanism of platinum resistance of these genes in ovarian cancer will be reported in the future.

244 Conclusions

245 In conclusion, we constructed a potential TF-miRNA-mRNA regulatory network related to 246 platinum resistance in ovarian cancer. It can help us identify some key genes and pathways 247 concerned with platinum resistance in ovarian cancer, which may assist in the treatment of 248 ovarian cancer and improve prognosis of ovarian cancer patients by targeting the established 249 miRNA-mRNA regulatory network in the future.

\section{Acknowledgment}

251 We thank Professor Li Bin from National Cancer Institute, Cancer Hospital, Chinese Academy 252 of Medical Sciences, Peking Union Medical College for their gifts of ovarian cancer cell lines. 
253

254

255

256

257

258

259

260

261

262

263

264

265

266

267

268

269

270

271

272

273

274

275

276

277

278

279

280

281

282

283

284

285

286

287

288

289

290

We also thank the GEO, DAVID, KEGG, STRING, miRNet and Cytoscape for providing their

platforms and the contributors for their valuable data sets.

\section{References}

Agarwal, R., and S. B. Kaye. 2003. 'Ovarian cancer: strategies for overcoming resistance to chemotherapy', Nat Rev Cancer, 3: 502-16.

Armstrong, D. K., R. D. Alvarez, J. N. Bakkum-Gamez, L. Barroilhet, K. Behbakht, A. Berchuck, L. M. Chen, M. Cristea, M. DeRosa, E. L. Eisenhauer, D. M. Gershenson, H. J. Gray, R. Grisham, A. Hakam, A. Jain, A. Karam, G. E. Konecny, C. A. Leath, J. Liu, H. Mahdi, L. Martin, D. Matei, M. McHale, K. McLean, D. S. Miller, D. M. O'Malley, S. Percac-Lima, E. Ratner, S. W. Remmenga, R. Vargas, T. L. Werner, E. Zsiros, J. L. Burns, and A. M. Engh. 2021. 'Ovarian Cancer, Version 2.2020, NCCN Clinical Practice Guidelines in Oncology', J Natl Compr Canc Netw, 19: 191-226.

Bai, T., Y. Zhao, Y. Liu, B. Cai, N. Dong, and B. Li. 2019. 'Effect of KNL1 on the proliferation and apoptosis of colorectal cancer cells', Technol Cancer Res Treat, 18: 1533033819858668.

Bie, L., G. Zhao, Y. P. Wang, and B. Zhang. 2012. 'Kinesin family member 2C (KIF2C/MCAK) is a novel marker for prognosis in human gliomas', Clin Neurol Neurosurg, 114: 356-60.

Cao, T., W. Pan, X. Sun, and H. Shen. 2019. 'Increased expression of TET3 predicts unfavorable prognosis in patients with ovarian cancer-a bioinformatics integrative analysis', J Ovarian Res, 12: 101.

Davidson, B., D. A. Nymoen, B. V. Elgaaen, A. C. Staff, C. G. Trope, J. Kaern, R. Reich, and T. E. Falkenthal. 2014. 'BUB1 mRNA is significantly co-expressed with AURKA and AURKB mRNA in advanced-stage ovarian serous carcinoma', Virchows Arch, 464: 701-7.

Dorayappan, K. D. P., R. Wanner, J. J. Wallbillich, U. Saini, R. Zingarelli, A. A. Suarez, D. E. Cohn, and K. Selvendiran. 2018. 'Hypoxia-induced exosomes contribute to a more aggressive and chemoresistant ovarian cancer phenotype: a novel mechanism linking STAT3/Rab proteins', Oncogene, 37: 3806-21.

Feng, H., Z. Y. Gu, Q. Li, Q. H. Liu, X. Y. Yang, and J. J. Zhang. 2019. 'Identification of significant genes with poor prognosis in ovarian cancer via bioinformatical analysis', J Ovarian Res, 12: 35.

Freimund, A. E., J. A. Beach, E. L. Christie, and D. D. L. Bowtell. 2018. 'Mechanisms of Drug Resistance in High-Grade Serous Ovarian Cancer', Hematol Oncol Clin North Am, 32: 983-96.

Gan, H., L. Lin, N. Hu, Y. Yang, Y. Gao, Y. Pei, K. Chen, and B. Sun. 2019. 'KIF2C exerts an oncogenic role in nonsmall cell lung cancer and is negatively regulated by miR-325-3p', Cell Biochem Funct, 37: 424-31.

Ghorbanhosseini, S. S., M. Nourbakhsh, M. Zangooei, Z. Abdolvahabi, Z. Bolandghamtpour, Z. Hesari, Z. Yousefi, G. Panahi, and R. Meshkani. 2019. 'MicroRNA-494 induces breast cancer cell apoptosis and reduces cell viability by inhibition of nicotinamide phosphoribosyltransferase expression and activity', EXCLI J, 18: 83851.

Gong, G., T. Lin, and Y. Yuan. 2020. 'Integrated analysis of gene expression and DNA methylation profiles in ovarian cancer', J Ovarian Res, 13: 30.

Gore, M. E., I. Fryatt, E. Wiltshaw, and T. Dawson. 1990. 'Treatment of relapsed carcinoma of the ovary with cisplatin or carboplatin following initial treatment with these compounds', Gynecol Oncol, 36: 207-11.

Han, J. Y., Y. K. Han, G. Y. Park, S. D. Kim, and C. G. Lee. 2015. 'Bub1 is required for maintaining cancer stem cells in

Peer) reviewing PDF | (2021:04:60745:1:1:NEW 12 Sep 2021) 
breast cancer cell lines', Sci Rep, 5: 15993.

He, H., X. Liao, Q. Yang, Y. Liu, Y. Peng, H. Zhong, J. Yang, H. Zhang, Z. Yu, Y. Zuo, C. Guan, and Z. Xu. 2018. 'MicroRNA-494-3p Promotes Cell Growth, Migration, and Invasion of Nasopharyngeal Carcinoma by Targeting Sox7', Technol Cancer Res Treat, 17: 1533033818809993.

Huo, Y., Z. Zheng, Y. Chen, Q. Wang, Z. Zhang, and H. Deng. 2016. 'Downregulation of vimentin expression increased drug resistance in ovarian cancer cells', Oncotarget, 7: 45876-88.

Ishikawa, K., Y. Kamohara, F. Tanaka, N. Haraguchi, K. Mimori, H. Inoue, and M. Mori. 2008. 'Mitotic centromereassociated kinesin is a novel marker for prognosis and lymph node metastasis in colorectal cancer', $\mathrm{Br} \mathrm{J}$ Cancer, 98: 1824-9.

Lee, Y. S., and A. Dutta. 2009. 'MicroRNAs in cancer', Annu Rev Pathol, 4: 199-227.

Li, T. F., H. J. Zeng, Z. Shan, R. Y. Ye, T. Y. Cheang, Y. J. Zhang, S. H. Lu, Q. Zhang, N. Shao, and Y. Lin. 2020. 'Overexpression of kinesin superfamily members as prognostic biomarkers of breast cancer', Cancer Cell Int, 20: 123.

Li, Y., and L. Li. 2019. 'Prognostic values and prospective pathway signaling of MicroRNA-182 in ovarian cancer: a study based on gene expression omnibus (GEO) and bioinformatics analysis', J Ovarian Res, 12: 106.

Liu, Y., X. Peng, H. Li, W. Jiao, X. Peng, J. Shao, Y. Xu, R. Wang, W. Wang, and D. Kong. 2021. 'STAT3 Inhibitor Napabucasin Inhibits Tumor Growth and Cooperates with Proteasome Inhibition in Human Ovarian Cancer Cells', Recent Pat Anticancer Drug Discov.

Lu, W., Y. Wu, C. X. Lu, T. Zhu, Z. L. Ren, and Z. Yu. 2020. 'Bioinformatics analysis of prognostic value and prospective pathway signal of miR-30a in ovarian cancer', J Ovarian Res, 13: 120.

Mo, Q. Q., P. B. Chen, X. Jin, Q. Chen, L. Tang, B. B. Wang, K. Z. Li, P. Wu, Y. Fang, S. X. Wang, J. F. Zhou, D. Ma, and G. Chen. 2013. 'Inhibition of Hec1 expression enhances the sensitivity of human ovarian cancer cells to paclitaxel', Acta Pharmacol Sin, 34: 541-8.

Nakamura, Y., F. Tanaka, N. Haraguchi, K. Mimori, T. Matsumoto, H. Inoue, K. Yanaga, and M. Mori. 2007. 'Clinicopathological and biological significance of mitotic centromere-associated kinesin overexpression in human gastric cancer', Br J Cancer, 97: 543-9.

Pan, X., Z. Guo, Y. Chen, S. Zheng, M. Peng, Y. Yang, and Z. Wang. 2020. 'STAT3-Induced IncRNA SNHG17 Exerts Oncogenic Effects on Ovarian Cancer through Regulating CDK6', Mol Ther Nucleic Acids, 22: 38-49.

Peng, Q. P., D. B. Du, Q. Ming, F. Hu, Z. B. Wu, and S. Qiu. 2018. 'MicroRNA 494 increases chemosensitivity to doxorubicin in gastric cancer cells by targeting phosphodiesterases 4D', Cell Mol Biol (Noisy-le-grand), 64: 62-66.

Piao, J., L. Zhu, J. Sun, N. Li, B. Dong, Y. Yang, and L. Chen. 2019. 'High expression of CDK1 and BUB1 predicts poor prognosis of pancreatic ductal adenocarcinoma', Gene, 701: 15-22.

Shen, P. F., X. Q. Chen, Y. C. Liao, N. Chen, Q. Zhou, Q. Wei, X. Li, J. Wang, and H. Zeng. 2014. 'MicroRNA-494-3p targets CXCR4 to suppress the proliferation, invasion, and migration of prostate cancer', Prostate, 74: 75667.

Tassi, R. A., A. Gambino, L. Ardighieri, E. Bignotti, P. Todeschini, C. Romani, L. Zanotti, M. Bugatti, F. Borella, D. Katsaros, G. Tognon, E. Sartori, F. Odicino, C. Romualdi, and A. Ravaggi. 2019. 'FXYD5 (Dysadherin) upregulation predicts shorter survival and reveals platinum resistance in high-grade serous ovarian cancer patients', Br J Cancer, 121: 584-92.

Wan, B. S., M. Cheng, and L. Zhang. 2019. 'Insulin-like growth factor 2 mRNA-binding protein 1 promotes cell 
332

333

334

335

336

337

338

339

340

341

342

343

344

345

346

347

348

349

350

351

352

353

354

355

356

357

358

359

360

361

362

363

364

365

366

367

368

369 proliferation via activation of AKT and is directly targeted by microRNA-494 in pancreatic cancer', World J Gastroenterol, 25: 6063-76.

Wang, D., and S. J. Lippard. 2005. 'Cellular processing of platinum anticancer drugs', Nat Rev Drug Discov, 4: 307-20. Wei, S., M. Dai, C. Zhang, K. Teng, F. Wang, H. Li, W. Sun, Z. Feng, T. Kang, X. Guan, R. Xu, M. Cai, and D. Xie. 2020. 'KIF2C: a novel link between Wnt/beta-catenin and mTORC1 signaling in the pathogenesis of hepatocellular carcinoma', Protein Cell.

Xu, X. H., S. J. Zhang, Q. B. Hu, X. Y. Song, and W. Pan. 2018. 'Effects of microRNA-494 on proliferation, migration, invasion, and apoptosis of medulloblastoma cells by mediating c-myc through the p38 MAPK signaling pathway', J Cell Biochem.

Yang, A., X. Wang, C. Yu, Z. Jin, L. Wei, J. Cao, Q. Wang, M. Zhang, L. Zhang, L. Zhang, and C. Hao. 2017. 'microRNA494 is a potential prognostic marker and inhibits cellular proliferation, migration and invasion by targeting SIRT1 in epithelial ovarian cancer', Oncol Lett, 14: 3177-84.

Yang, D., Y. He, B. Wu, Y. Deng, N. Wang, M. Li, and Y. Liu. 2020. 'Integrated bioinformatics analysis for the screening of hub genes and therapeutic drugs in ovarian cancer', J Ovarian Res, 13: 10.

Yu, F., P. Qiao, G. Yin, Y. Sun, X. Yu, X. Sun, Y. Chu, and Y. Wang. 2020. 'RHPN2 Promotes Malignant Cell Behaviours in Ovarian Cancer by Activating STAT3 Signalling', Onco Targets Ther, 13: 11517-27.

Zhang, F., J. Y. Cui, H. F. Gao, H. Yu, F. F. Gao, J. L. Chen, and L. Chen. 2020. 'Cancer-associated fibroblasts induce epithelial-mesenchymal transition and cisplatin resistance in ovarian cancer via CXCL12/CXCR4 axis', Future Oncol, 16: 2619-33.

Zhang, L., L. Sun, B. Zhang, and L. Chen. 2019. 'Identification of Differentially Expressed Genes (DEGs) Relevant to Prognosis of Ovarian Cancer by Use of Integrated Bioinformatics Analysis and Validation by Immunohistochemistry Assay', Med Sci Monit, 25: 9902-12.

Zhang, Y., S. Chen, C. Wei, G. O. Rankin, X. Ye, and Y. C. Chen. 2018. 'Flavonoids from Chinese bayberry leaves induced apoptosis and G1 cell cycle arrest via Erk pathway in ovarian cancer cells', Eur J Med Chem, 147: 218-26.

Zhang, Y., L. Guo, Y. Li, G. H. Feng, F. Teng, W. Li, and Q. Zhou. 2018. 'MicroRNA-494 promotes cancer progression and targets adenomatous polyposis coli in colorectal cancer', Mol Cancer, 17: 1.

Zhao, F., M. K. Siu, L. Jiang, K. F. Tam, H. Y. Ngan, X. F. Le, O. G. Wong, E. S. Wong, A. R. Gomes, L. Bella, P. Khongkow, E. W. Lam, and A. N. Cheung. 2014. 'Overexpression of forkhead box protein M1 (FOXM1) in ovarian cancer correlates with poor patient survival and contributes to paclitaxel resistance', PLoS One, 9: e113478.

Zheng, M. J., X. Li, Y. X. Hu, H. Dong, R. Gou, X. Nie, Q. Liu, H. Ying-Ying, J. J. Liu, and B. Lin. 2019. 'Identification of molecular marker associated with ovarian cancer prognosis using bioinformatics analysis and experiments', J Cell Physiol, 234: 11023-36.

Zhong, Y., F. Le, J. Cheng, C. Luo, X. Zhang, X. Wu, F. Xu, Q. Zuo, and B. Tan. 2021. 'Triptolide inhibits JAK2/STAT3 signaling and induces lethal autophagy through ROS generation in cisplatinresistant SKOV3/DDP ovarian cancer cells', Oncol Rep, 45. 


\section{Table $\mathbf{1}$ (on next page)}

Nucleotide sequences of the primers 
1 Table 1 Nucleotide sequences of the primers

\begin{tabular}{lll}
\hline Gene name & Forward primer $\left(5^{\prime}\right.$ to $3^{\prime}$ ) & Reverse primer $\left(5^{\prime}\right.$ to $3^{\prime}$ ) \\
\hline NUF2 & GGAAGGCTTCTTACCATTCAGC & GACTTGTCCGTTTTGCTTTTGG \\
CCNB2 & CCGACGGTGTCCAGTGATTT & TGTTGTTTTGGTGGGTTGAACT \\
CENPN & TGAACTGACAACAATCCTGAAGG & CTTGCACGCTTTTCCTCACAC \\
KIF2C & CTGTTTCCCGGTCTCGCTATC & AGAAGCTGTAAGAGTTCTGGGT \\
NUP43 & TGCCTCCGGGAAGTTTACAGA & TCTCCTTCAAACCCTCCATCA \\
NDC80 & CCTCTCCATGCAGGAGTTAAGA & GGTCTCGGGTCCTTGATTTTCT \\
BUB1 & TGGGAAAGATACATACAGTGGGT & AGGGGATGACAGGGTTCCAAT \\
GAPDH & GGAGCGAGATCCCTCCAAAAT & GGCTGTTGTCATACTTCTCATGG \\
\hline
\end{tabular}

2 


\section{Table 2 (on next page)}

Informationabout the DEGs identified from the datasets

A total of 187 DEGs were identified from GSE 28739, including 63 upregulated DEGs and 124 downregulated DEGs in platinum-resistant ovarian cancer samples compared to platinum-sensitive samples. 
Table 2 Information about the DEGs identified from the datasets

\begin{tabular}{|c|c|}
\hline DEGs & Gene name \\
\hline $\begin{array}{l}\text { Upregulated } \\
\text { (63) }\end{array}$ & $\begin{array}{l}\text { THEMIS2, ACADVL, EGR2, HBA2, HBB, HBA1, HBG1, IL1B, COMT, PTGDS, } \\
\text { EVI2A, SREBF1, INPP5D, EHBP1L1, ARHGDIB, IL10RA, GRB2, IGFBP6, } \\
\text { PLEK, CCL2, HIRA, SOCS3, FCGR2B, PLK3, CD163, ARHGDIA, DUSP2, } \\
\text { DUSP5, OVOL1, CEBPB, CD164, NNMT, SMPDL3A, LAPTM5, PNRC1, IFI44L, } \\
\text { VSIG4, CDC42EP4, DPP7, MICAL2, SWAP70, STAB1, ABI3BP, CPAMD8, } \\
\text { RMDN1, FCGR2A, DIDO1, IFI6, METRN, NFKBIZ, GNPTG, HAVCR2, CARD9, } \\
\text { CMTM7, SCEL, OSR1, ZNF385B, COG7, CCDC117, C1orf162, ARID5A, STAT3, } \\
\text { MALAT1 }\end{array}$ \\
\hline $\begin{array}{l}\text { Downregulated } \\
\text { (124) }\end{array}$ & $\begin{array}{l}\text { FBXW4P1, RBM33, SLC25A16, MSH6, MID1, GSTP1, SSBP3, LAMB3, MYO19, } \\
\text { C2orf88, ZBTB38, ASRGL1, GBAS, ASNS, CKS2, COX7B, HSPE1, EPCAM, } \\
\text { NDUFB3, POLA2, POU2F1, SCG5, SNRPE, SRP19, SSBP1, ZNF45, HIST1H4I, } \\
\text { HIST1H2BN, HIST1H2BE, HIST1H2BC, HIST1H4F, HIST1H4C, HIST1H4H, } \\
\text { HIST1H4B, HIST1H4L, LGR5, STC2, MPZL1, FKBP5, BUB1, COX6A1, GCSH, } \\
\text { NDUFA1, PSPH, CCNB2, C14orf2, SFPQ, HINT1, LAMC2, SMO, TCEB1, } \\
\text { RABEPK, POP7, PRSS16, ACAT2, NDC80, SIVA1, EBNA1BP2, KIF2C, PYCR1, } \\
\text { MYCBP, PRDM4, H2AFV, DNAJC15, HILPDA, METTL5, CRIPT, CNIH4, } \\
\text { SEC61G, FAM162A, MELK, ZNF777, TRIM24, MRPS17, MRPS33, SCFD1, } \\
\text { CHCHD2, TRIAP1, RSRC1, WAC, FOLR1, F11R, C1orf109, YEATS2, STYK1, } \\
\text { CENPN, LRRC59, ZNF395, PCDHB2, EML4, SPC25, SYT13, ZNF14, RAB38, } \\
\text { SOX17, TMEM106C, TRAPPC6A, UBL5, CDCA7, AJUBA, PURB, SIGLEC1,, } \\
\text { TSEN15, PSAT1, MACROD2, NSMCE3, TRUB1, CIART, NUF2, GSTA1, SGPP2, } \\
\text { CDCA2, ZNF48, TMEM139, GSTA5, NASP, ERICH5, TMEM136, PTPMT1, } \\
\text { RFC5, GOLT1A, NUP43, FGF7P6, TP53TG1 }\end{array}$ \\
\hline
\end{tabular}

2 A total of 187 DEGs were identified from GSE 28739, including 63 upregulated DEGs and 124 downregulated DEGs in 3 platinum-resistant ovarian cancer samples compared to platinum-sensitive samples. 


\section{Table 3(on next page)}

Table 3 Information aboutthe DE-miRNAs identified from the datasets

A total of 10 DE-miRNAs were identified from GSE 25202, including 4 upregulated miRNAs and 6 downregulated miRNAs in platinum-resistant ovarian cancer samples compared to platinum-sensitive samples. 
1 Table 3 Information about the DE-miRNAs identified from the datasets

\begin{tabular}{ll}
\hline DE-miRNAs & MiRNA name \\
\hline Upregulated & hsa-miR-656, hsa-miR-655, hsa-miR-379*, hsa-miR-494 \\
(4) & hsa-miR-506, hsa-miR-513a-3p, hsa-miR-513c, hsa-miR-510, hsa-miR-509-3-5p, \\
Downregulated & hsa-miR-202*:9.1 \\
(6)
\end{tabular}

2 A total of 10 DE-miRNAs were identified from GSE 25202, including 4 upregulated miRNAs and 6 downregulated miRNAs in

3 platinum-resistant ovarian cancer samples compared to platinum-sensitive samples. 


\section{Table 4(on next page)}

Table 4 Theinformation about the top ten hub genes.

Table 4 The information about the top ten hub genes. 
1 Table 4 The information about the top ten hub genes.

\begin{tabular}{llc}
\hline Gene symbol & Gene description & Score \\
\hline NUF2 & NDC80 kinetochore complex component & 9 \\
CCNB2 & cyclin B2 & 9 \\
CENPN & centromere protein N & 9 \\
BUB1 & BUB1 mitotic checkpoint serine/threonine kinase & 8 \\
NDC80 & NDC80, kinetochore complex component & 8 \\
NUP43 & nucleoporin 43 & 8 \\
KIF2C & kinesin family member 2C & 8 \\
STAT3 & signal transducer and activator of transcription 3 & 8 \\
HIST1H2BE & histone cluster 1 H2B family member e & 8 \\
HIST1H2BC & histone cluster 1 H2B family member c & 7 \\
\hline
\end{tabular}

2 
Figure 1

Volcano plot of differentially expressed genes (DEGs). Red: upregulated genes;Green: downregulated genes.

Figure 1 Volcano plot of differentially expressed genes (DEGs). Red: upregulated genes; Green: downregulated genes.

\section{GSE28739 resistant vs sensitive}

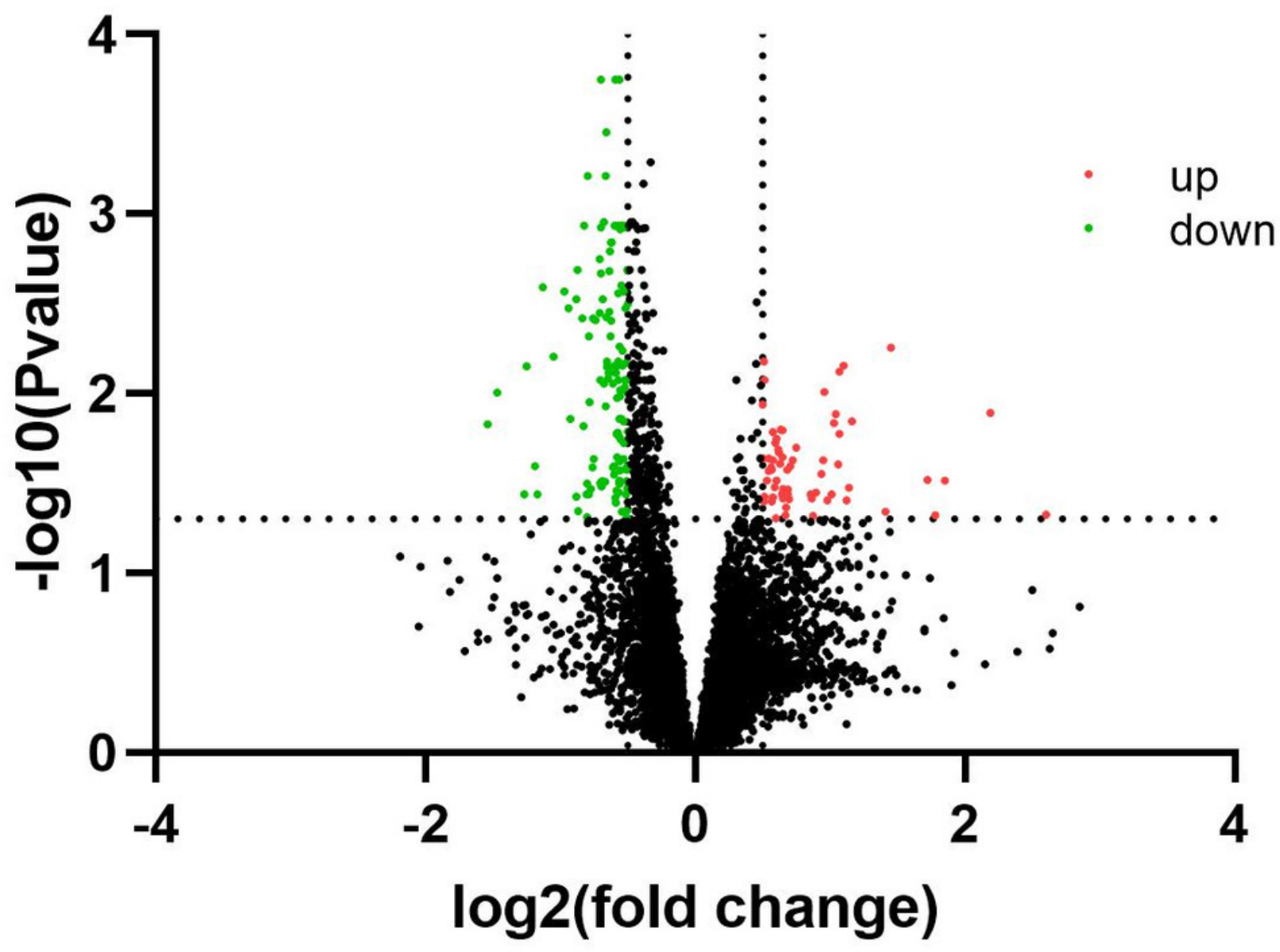


Figure 2

Volcano plot of differentially expressed miRNAs (DE-miRNAs). Red: upregulated miRNAs;Green: downregulated miRNAs.

Figure 2 Volcano plot of differentially expressed miRNAs (DE-miRNAs). Red: upregulated miRNAs; Green: downregulated miRNAs.

\section{GSE25202 resistant vs sensitive}

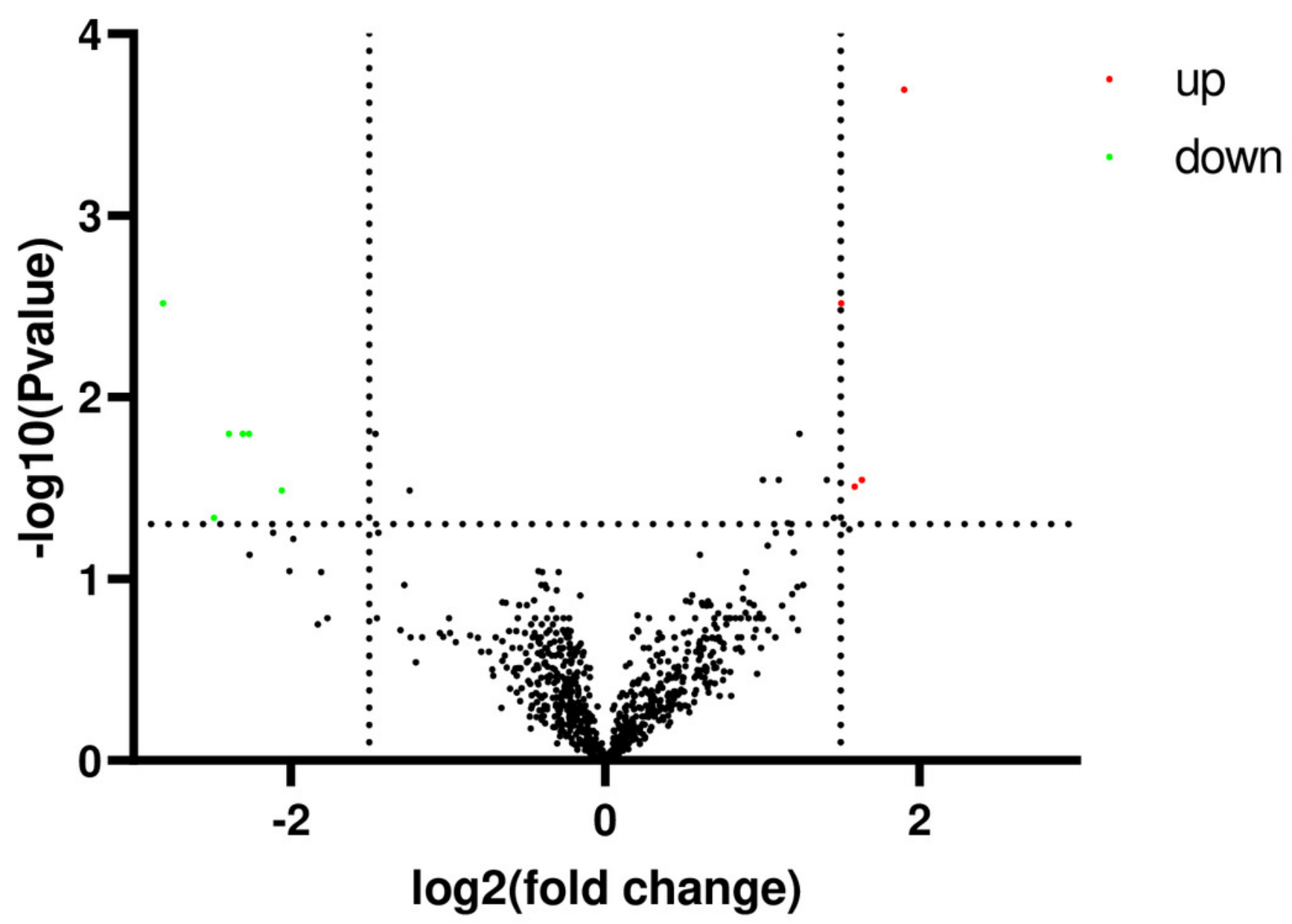


Figure 3

Figure 3 Geneontology (GO) enrichment analysis.

Figure 3 Gene ontology (GO) enrichment analysis.

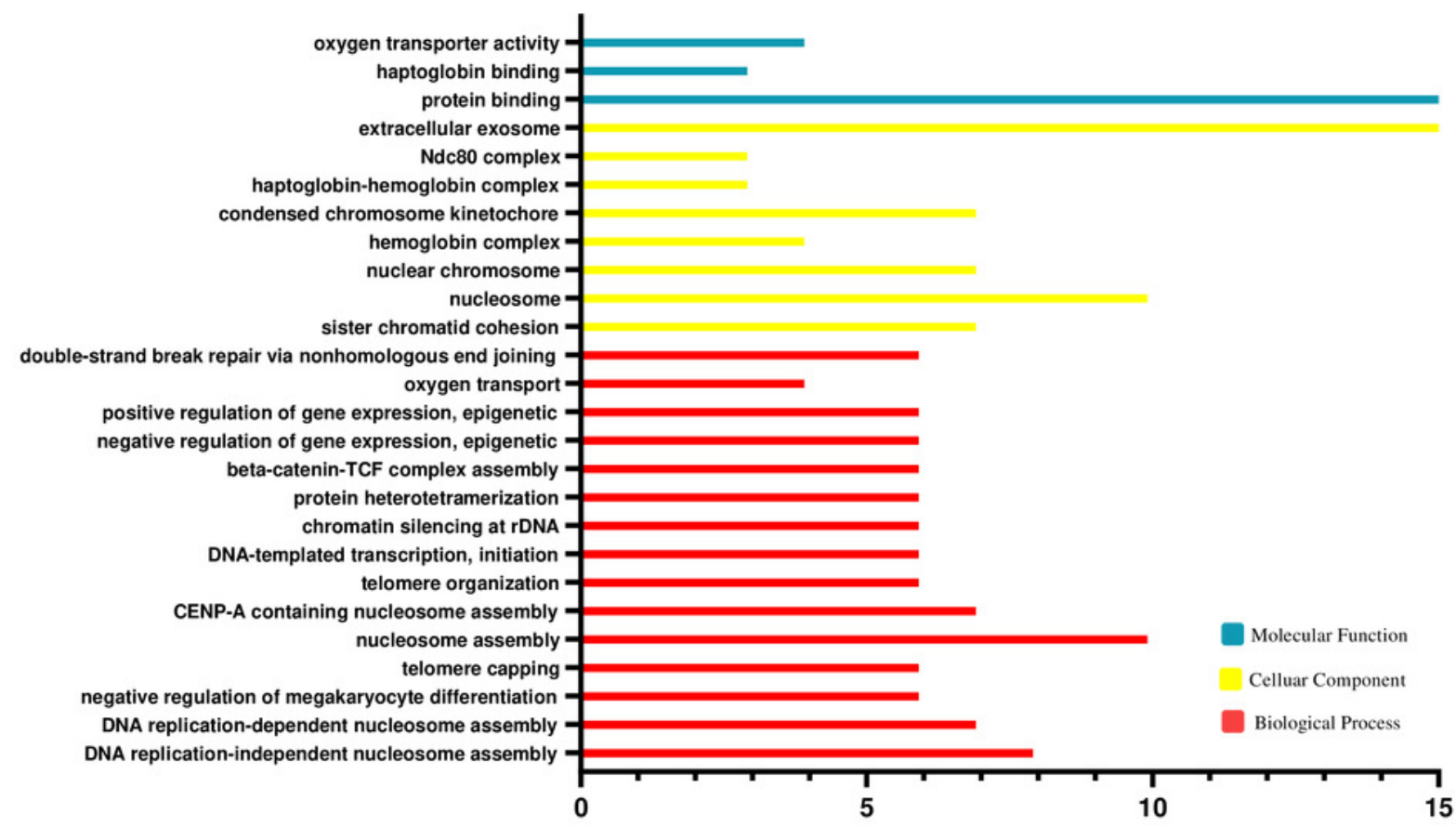


Figure 4

Figure 4 Bubble map for Kyoto Encyclopedia of Genes andGenomes (KEGG) Pathway Analysis of DEGs.

Figure 4 Bubble map for Kyoto Encyclopedia of Genes and Genomes (KEGG) Pathway Analysis of DEGs.

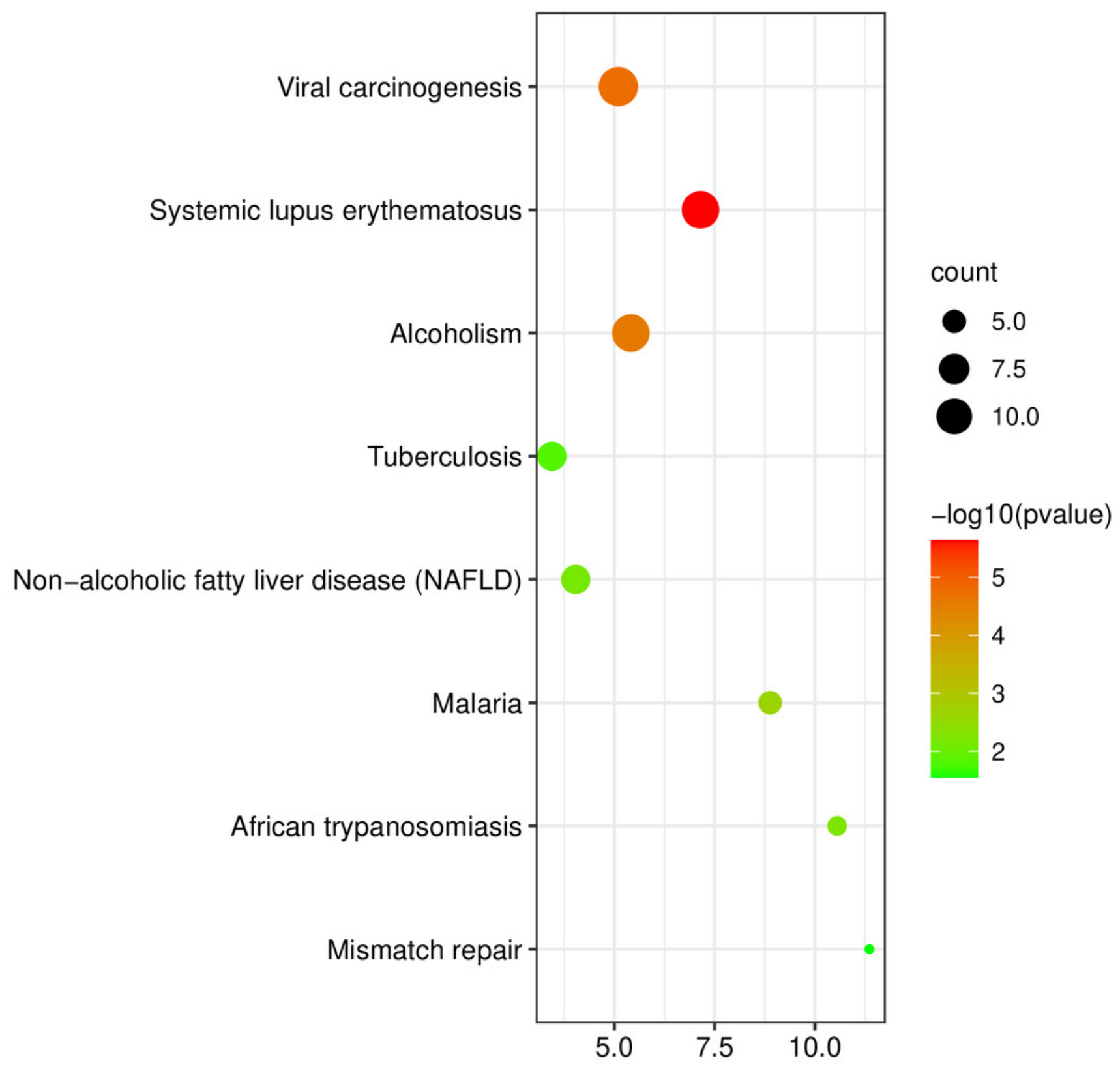




\section{Figure 5}

Figure 5 Protein-proteininteraction network of differentially expressed genes (DEGs). Circles representgenes, lines represent the interaction of proteins between genes. Yellow: upregulated genes; Blue:downregulated genes.

Figure 5 Protein-protein interaction network of differentially expressed genes (DEGs). Circles represent genes, lines represent the interaction of proteins between genes. Yellow: upregulated genes; Blue: downregulated genes. 


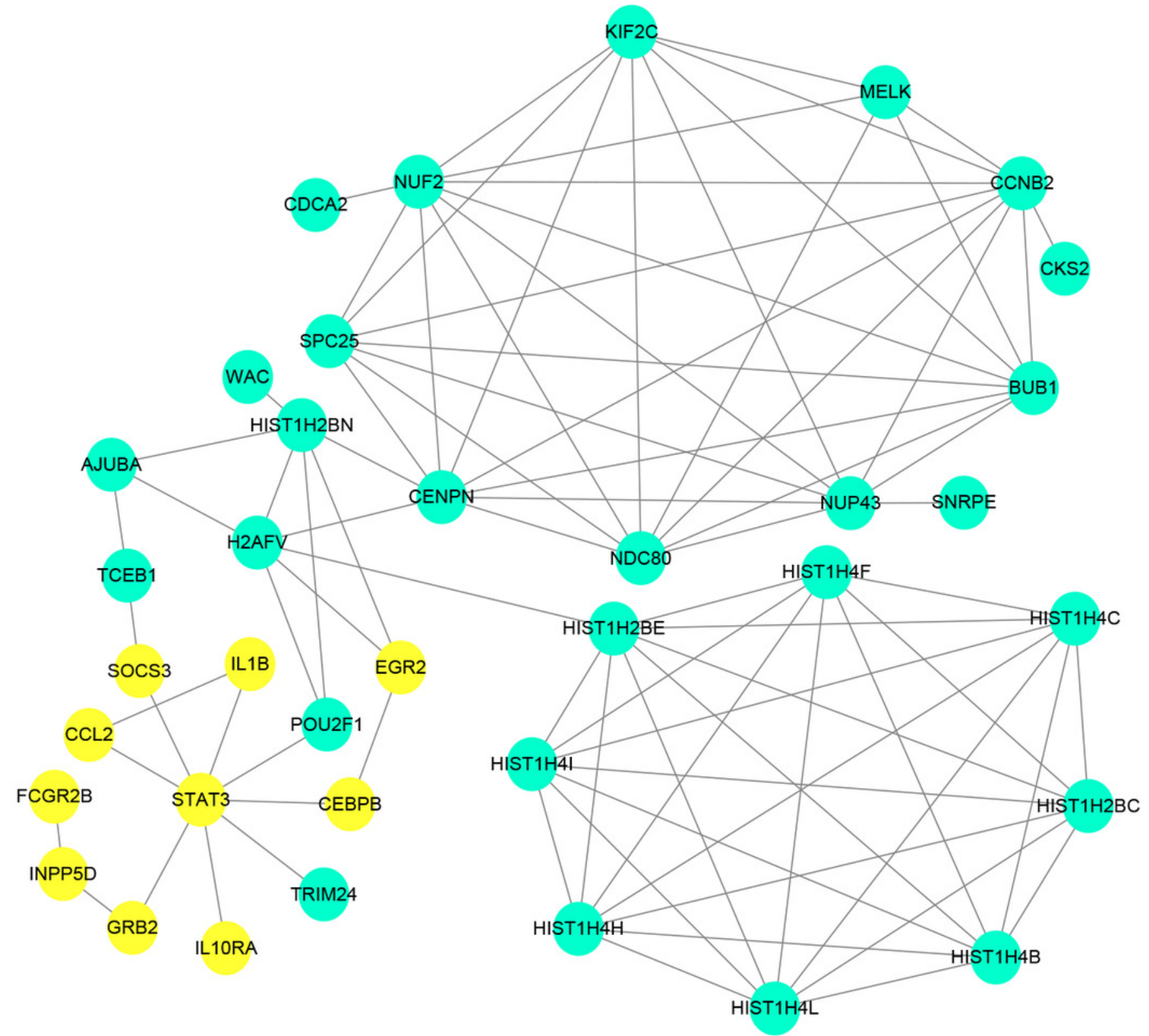


Figure 6

Figure 6 Theinteraction network of the hub genes and their related genes.

Figure 6 The interaction network of the hub genes and their related genes.

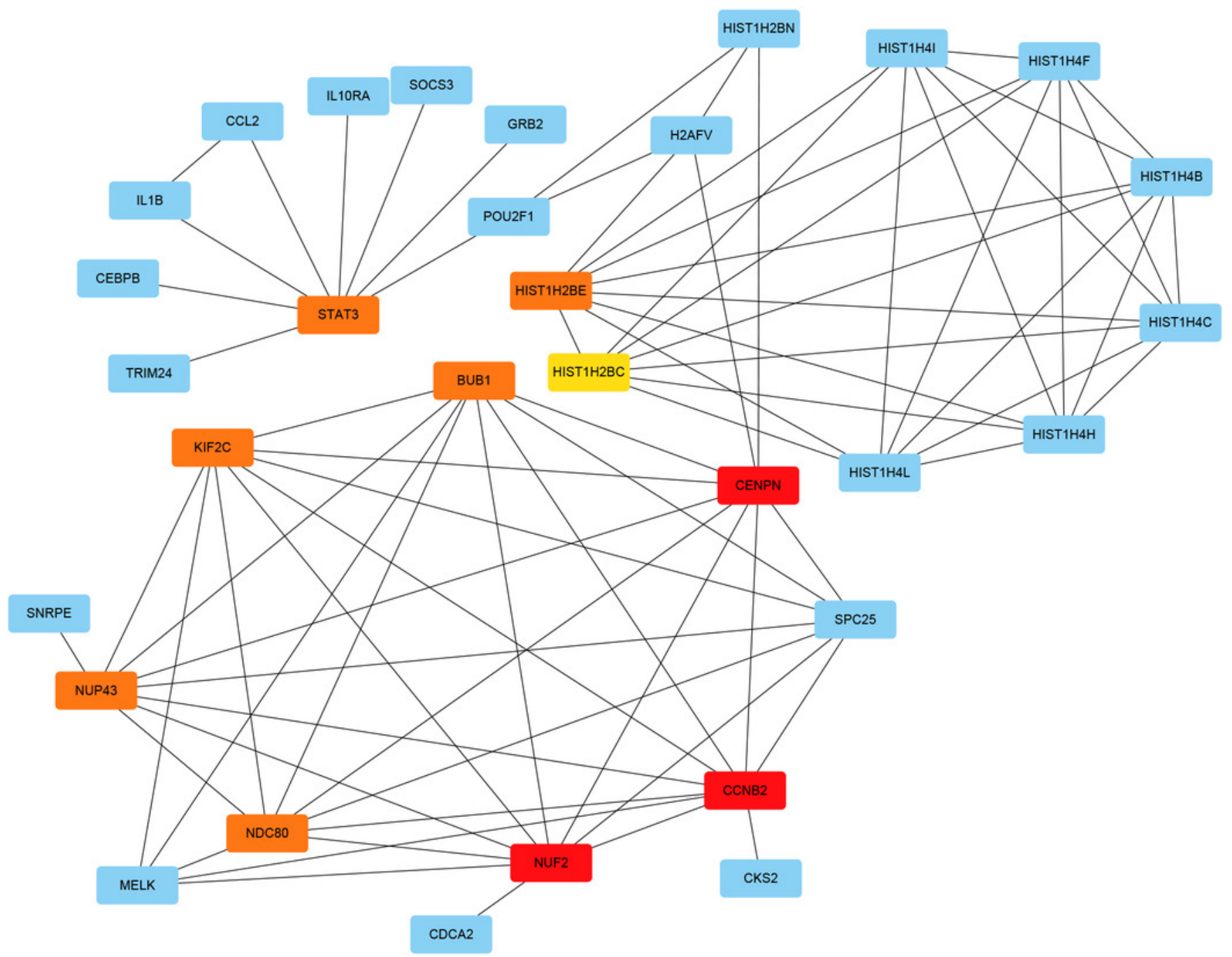


Figure 7

Figure 7 The hub genes with the highest degree scores of connectivity in the PPInetwork.

Figure 7 The hub genes with the highest degree scores of connectivity in the PPI network.

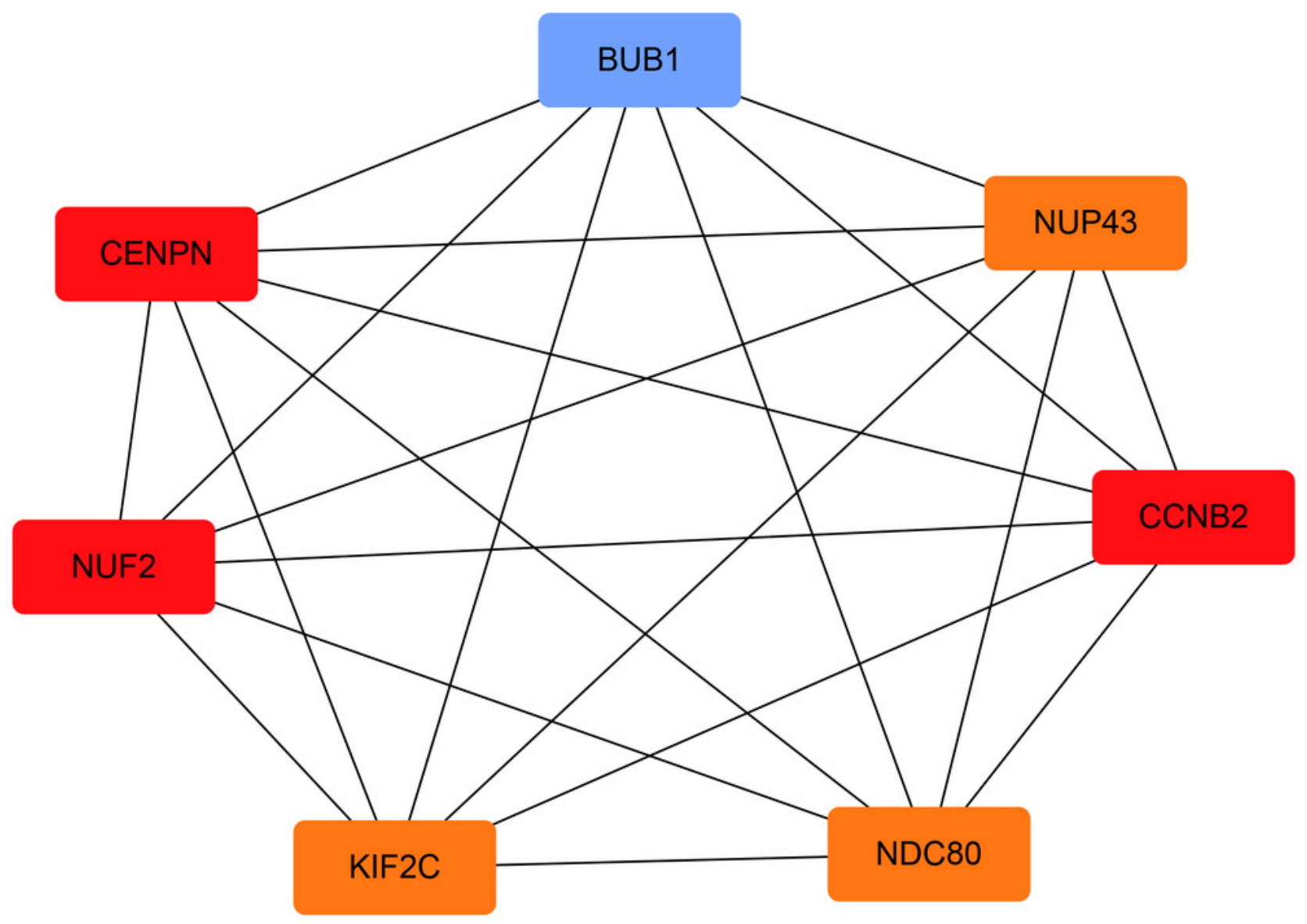

HIST1H2BC 
Figure 8

Figure 8 QRT-PCR analysis of BUB1,KIF2C, NUP43, NDC80, NUF2, CCNB2 and CENPN in A2780 cis-platinum resistant celllines compared with A2780 cell line.

Figure 8 QRT-PCR analysis of BUB1, KIF2C, NUP43, NDC80, NUF2, CCNB2 and CENPN in A2780 cis-platinum resistant cell lines compared with A2780 cell line.

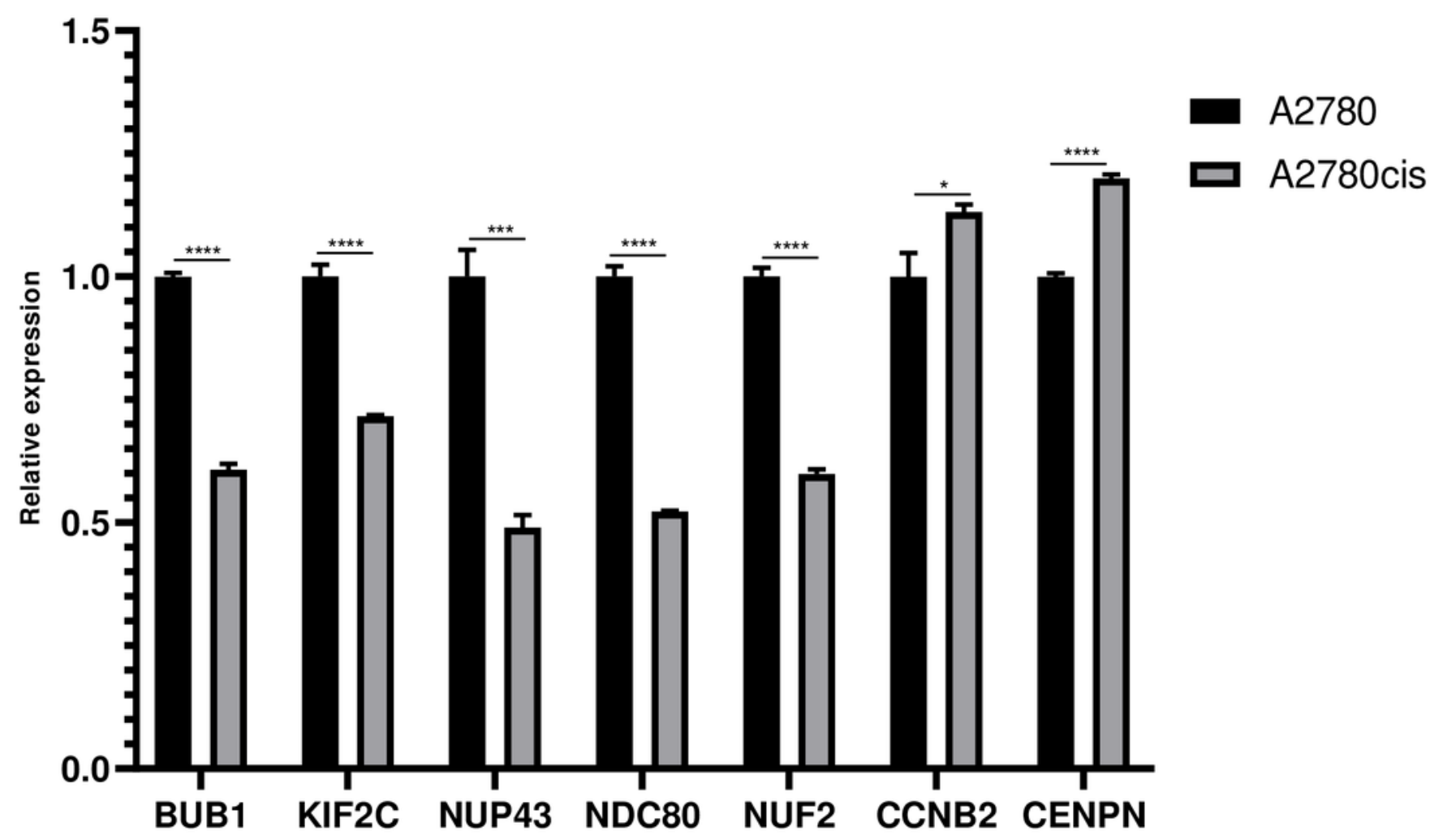




\section{Figure 9}

Figure 9 Transcriptionfactors of upregulated miRNAs.

Figure 9 Transcription factors of upregulated miRNAs. 


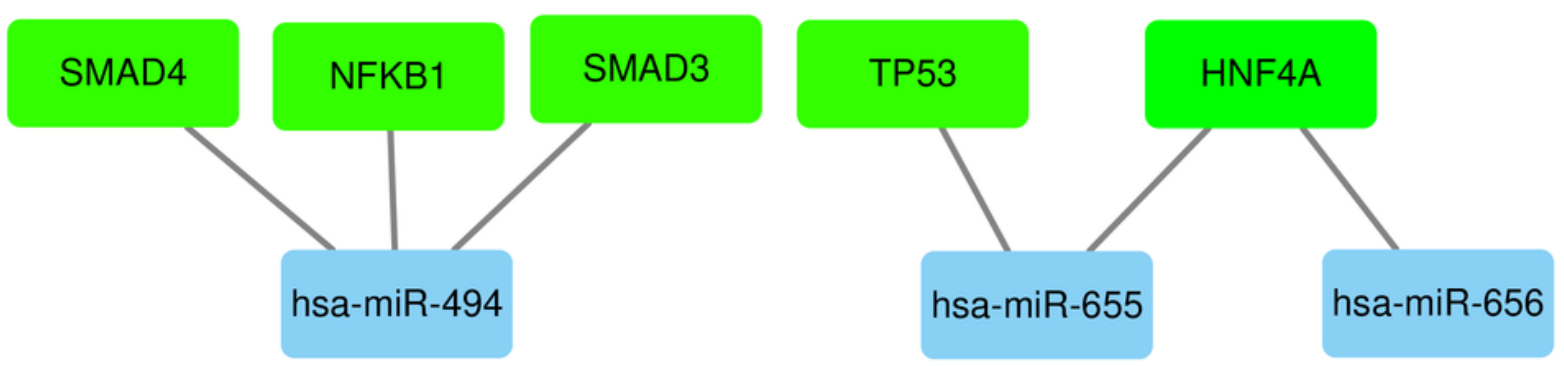




\section{Figure 10}

Figure $\mathbf{1 0}$ The miRNA-target gene regulatory network.

Figure $\mathbf{1 0}$ The miRNA-target gene regulatory network. 


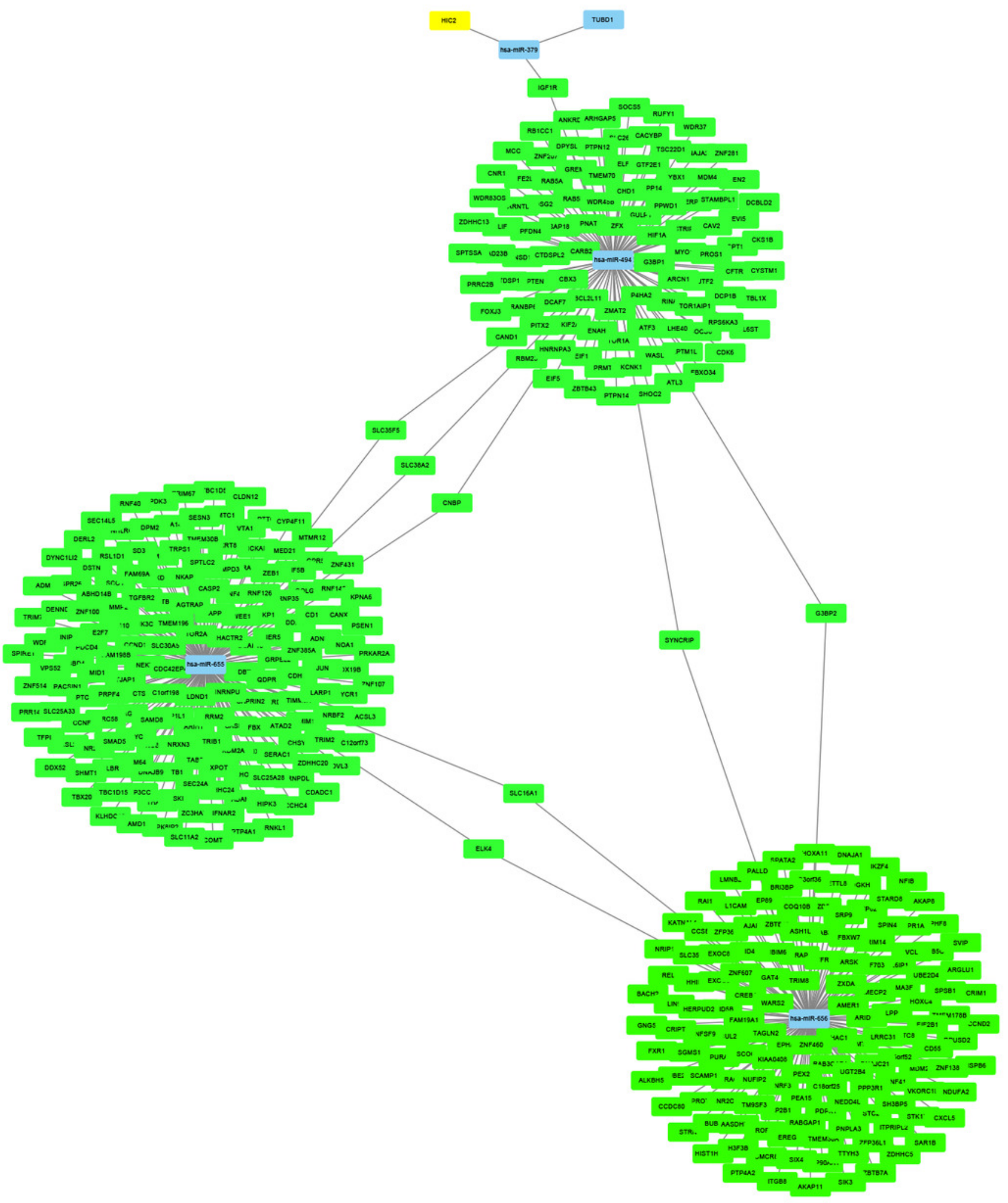

